\title{
Computer-assisted planning for the insertion of stereoelectroencephalography electrodes for the investigation of drug-resistant focal epilepsy: an external validation study
}

\author{
Vejay N. Vakharia, MRCS, ${ }^{1-3}$ Rachel Sparks, PhD, ${ }^{4}$ Roman Rodionov, PhD, ${ }^{1-3}$ Sjoerd B. Vos, PhD, ${ }^{2,4}$ \\ Christian Dorfer, MD, ${ }^{5}$ Jonathan Miller, MD, ${ }^{6}$ Daniel Nilsson, MD, ${ }^{7}$ Martin Tisdall, MD, ${ }^{8}$ Stefan \\ Wolfsberger, MD, ${ }^{5}$ Andrew W. McEvoy, FRCS(SN), ${ }^{1,3}$ Anna Miserocchi, MD, ${ }^{1}$ Gavin P. Winston, \\ $\mathrm{PhD},{ }^{1-3}$ Aidan G. O'Keeffe, PhD, ${ }^{9}$ Sebastien Ourselin, PhD, ${ }^{1,4}$ and John S. Duncan, FRCP ${ }^{1-3}$ \\ 'Department of Clinical and Experimental Epilepsy, UCL Institute of Neurology, and ${ }^{3}$ Department of Neurosurgery, National Hospital \\ for Neurology and Neurosurgery, Queen Square, London; ${ }^{4}$ Transitional Imaging Group, Centre for Medical Image Computing, and \\ ${ }^{9}$ Department of Statistical Science, University College London; ${ }^{8}$ Great Ormond Street Hospital, UCL Great Ormond Street Institute \\ of Child Health, London; ' ${ }^{2}$ Epilepsy Society MRI Unit, Chalfont St Peter, United Kingdom; ${ }^{5}$ Department of Neurosurgery, Medical \\ University Vienna, General Hospital (AKH) Waehringer Guertel, Vienna, Austria; ${ }^{6}$ Department of Neurological Surgery, University \\ Hospitals Cleveland Medical Center, Case Western Reserve University School of Medicine, Cleveland, Ohio; and ${ }^{7}$ Institute of \\ Neuroscience and Physiology, Sahlgrenska Academy, Gothenburg University, Göteborg, Sweden
}

OBJECTIVE One-third of cases of focal epilepsy are drug refractory, and surgery might provide a cure. Seizure-free outcome after surgery depends on the correct identification and resection of the epileptogenic zone. In patients with no visible abnormality on MRI, or in cases in which presurgical evaluation yields discordant data, invasive stereoelectroencephalography (SEEG) recordings might be necessary. SEEG is a procedure in which multiple electrodes are placed stereotactically in key targets within the brain to record interictal and ictal electrophysiological activity. Correlating this activity with seizure semiology enables identification of the seizure-onset zone and key structures within the ictal network. The main risk related to electrode placement is hemorrhage, which occurs in $1 \%$ of patients who undergo the procedure. Planning safe electrode placement for SEEG requires meticulous adherence to the following: 1) maximize the distance from cerebral vasculature, 2) avoid crossing sulcal pial boundaries (sulci), 3) maximize gray matter sampling, 4) minimize electrode length, 5) drill at an angle orthogonal to the skull, and 6) avoid critical neurological structures. The authors provide a validation of surgical strategizing and planning with EpiNav, a multimodal platform that enables automated computer-assisted planning (CAP) for electrode placement with user-defined regions of interest.

METHODS Thirteen consecutive patients who underwent implantation of a total 116 electrodes over a 15-month period were studied retrospectively. Models of the cortex, gray matter, and sulci were generated from patient-specific wholebrain parcellation, and vascular segmentation was performed on the basis of preoperative MR venography. Then, the multidisciplinary implantation strategy and precise trajectory planning were reconstructed using CAP and compared with the implemented manually determined plans. Paired results for safety metric comparisons were available for 104 electrodes. External validity of the suitability and safety of electrode entry points, trajectories, and target-point feasibility was sought from 5 independent, blinded experts from outside institutions.

RESULTS CAP-generated electrode trajectories resulted in a statistically significant improvement in electrode length, drilling angle, gray matter-sampling ratio, minimum distance from segmented vasculature, and risk $(p<0.05)$. The

ABBREVIATIONS CAP = computer-assisted planning; DBS = deep brain stimulation; DSA = digital subtraction angiography; EEG = electroencephalography; EZ = epileptogenic zone; FOV = field of view; MDT = multidisciplinary team; MRA = MR angiography; MRV = MR venography; ROI = region of interest; SEEG = stereoelectroencephalography.

SUBMITTED July 26, 2017. ACCEPTED October 2, 2017

INCLUDE WHEN CITING Published online April 13, 2018; DOI: 10.3171/2017.10.JNS171826. 
blinded external raters had various opinions of trajectory feasibility that were not statistically significant, and they considered a mean of $69.4 \%$ of manually determined trajectories and $62.2 \%$ of CAP-generated trajectories feasible; $19.4 \%$ of the CAP-generated electrode-placement plans were deemed feasible when the manually determined plans were not, whereas $26.5 \%$ of the manually determined electrode-placement plans were rated feasible when CAP-determined plans were not (no significant difference).

CONCLUSIONS CAP generates clinically feasible electrode-placement plans and results in statistically improved safety metrics. CAP is a useful tool for automating the placement of electrodes for SEEG; however, it requires the operating surgeon to review the results before implantation, because only $62 \%$ of electrode-placement plans were rated feasible, compared with $69 \%$ of the manually determined placement plans, mainly because of proximity of the electrodes to unsegmented vasculature. Improved vascular segmentation and sulcal modeling could lead to further improvements in the feasibility of CAP-generated trajectories.

https://thejns.org/doi/abs/10.3171/2017.10.JNS171826

KEYWORDS stereoelectroencephalography; computer-assisted planning; epilepsy evaluation; external validation; EpiNav

$\mathrm{E}$ PILEPSY has been defined as "a disorder of the brain characterized by an enduring predisposition to generate epileptic seizures."10 Epilepsy can have wide-ranging effects on a patient's quality of life and can result in physical injury, psychosocial dysfunction, cognitive decline, and risk of death. ${ }^{14}$ One-third of patients with epilepsy continue to have seizures despite their use of 2 or more appropriately prescribed antiepileptic drug schedules. These patients are defined as having drugresistant epilepsy. ${ }^{25}$ Surgical intervention can potentially cure drug-resistant epilepsy if the region from which the seizures arise, known as the epileptogenic zone (EZ), can be identified and removed safely. A patient's chances of achieving sustained freedom from seizures after epilepsy surgery are highest when the seizure semiology, electrophysiological investigations, imaging findings, and neuropsychological assessment are concordant. In such cases, the patient does not require any further imaging or testing unless there is proximity of the suspected EZ to eloquent cortex and resective surgery can be performed. In a proportion of patients, results of the noninvasive presurgical evaluation are not clear or discordant, and invasive intracranial EEG recordings, in the form of either grid/strip implantation or stereoelectroencephalography (SEEG), are required. SEEG involves the stereotactic placement of multiple (8-16) electrodes at predefined regions of the brain to help delineate the EZ and the spatial and temporal seizure-network spread within the brain. A recent meta-analysis regarding the safety of electrode implantation for SEEG found the overall risk of complications to be $1.3 \%$ per patient. The greatest risk related to electrode placement is intracranial hemorrhage, which had a pooled prevalence of $1 \%$ per patient. ${ }^{15}$ The factors that determine the risk of hemorrhage are the initial planned trajectory and the accuracy of the implantation method. The methods currently used to implant electrodes for SEEG involve stereotactic frame-based, frameless, and robotic systems. There is a paucity of evidence in the literature from comparisons of these methods performed to determine which one is the most accurate, but entry and target point accuracies have ranged from 0.78 to 3.5 and 1.70 to $3.66 \mathrm{~mm}$, respectively. ${ }^{28}$

Electrode trajectories currently are planned manually to sample the regions of interest (ROIs) while maximiz- ing gray matter contact and distance from blood vessels. This task is time-consuming and requires significant multidisciplinary input. We previously described the benefits of multimodal 3D imaging for manual electrode planning and an early version of computer-assisted planning (CAP). ${ }^{17,18}$ In the initial study, manually planned electrodeimplantation schemes for 18 patients (166 electrodes) were recreated retrospectively using EpiNav software. An earlier version of the software required the target points for the electrodes to be placed manually on the MR image, and the software then would calculate the safest electrode trajectory based on the cumulative distance from segmented blood vessels along the whole trajectory. ${ }^{18}$ The computer-generated and manually determined trajectories then were rated by 3 independent, blinded neurosurgeons as to whether they were feasible for implantation. Overall, the computer-generated electrodes resulted in significantly shorter intracranial length, increased distance from blood vessels, greater gray matter sampling, and improved drilling angles $(\mathrm{p}<0.05$ for all parameters). Of the computer-generated electrodes, $78.9 \%$ were deemed feasible for implantation by at least 2 of the 3 independent neurosurgeons.

Further development of the EpiNav software implemented its ability to define entry and target zones constrained by anatomical structures. ${ }^{24}$ Users can now define an ROI by typing or clicking on an anatomical location (e.g., right amygdala) and allowing the computer algorithm to define the safest entry and target points within the anatomical structure as a whole. Furthermore, multiple trajectories can be placed within the same anatomical structure, and electrodes will be spread evenly within safe zones to maximize region sampling. This ability is of particular benefit for large anatomical targets, such as the cingulate cortex, and when high-density sampling of a structure such as the insula or hippocampus is required. We confirmed external validity of the generated electrodes from 5 independent, blinded epilepsy neurosurgeons, from outside institutions, who had expertise in implanting electrodes for SEEG and none of whom were involved in generation of the initial manually determined plans. To gauge surgeon variability and preferences, we assessed why surgeons rated trajectories as infeasible. The implantation methods used by the external raters included frame- 
TABLE 1. Patient demographics

\begin{tabular}{|c|c|c|c|c|c|c|}
\hline $\begin{array}{l}\text { Case } \\
\text { No. }\end{array}$ & $\begin{array}{l}\text { Age at Electrode } \\
\text { Implantation (yrs) }\end{array}$ & Sex & $\begin{array}{l}\text { Implanted } \\
\text { Hemisphere }\end{array}$ & $\begin{array}{l}\text { Hemispheric Language } \\
\text { Dominance (fMRI) }\end{array}$ & Hypothesized EZ & $\begin{array}{c}\text { No. of } \\
\text { Electrodes }\end{array}$ \\
\hline 1 & 37 & M & Lt & $\mathrm{Lt}$ & Lt frontal (nonlesional) & 11 \\
\hline 2 & 27 & M & Rt & $\mathrm{Lt}$ & Rt frontal (nonlesional) & 8 \\
\hline 3 & 45 & $\mathrm{~F}$ & Rt & Bilat & Rt frontal (nonlesional) & 13 \\
\hline 4 & 35 & $\mathrm{~F}$ & $\mathrm{Lt}$ & Lt & Lt temporal (lesional) & 3 \\
\hline 5 & 31 & M & $\mathrm{Rt}$ & $\mathrm{Lt}$ & Rt temporal (lesional) & 8 \\
\hline 6 & 42 & M & Rt & Lt & Rt frontal (nonlesional) & 10 \\
\hline 7 & 49 & $\mathrm{~F}$ & Rt & $\mathrm{Lt}$ & Rt temporal (nonlesional) & 10 \\
\hline 8 & 61 & M & Rt & $\mathrm{Lt}$ & Rt frontal (nonlesional) & 11 \\
\hline 9 & 24 & M & Rt & $\mathrm{Lt}$ & Rt frontal (nonlesional) & 8 \\
\hline 10 & 42 & M & $\mathrm{Lt}$ & $\mathrm{Lt}$ & Lt frontal (nonlesional) & 6 \\
\hline 11 & 31 & M & Bilat & $\mathrm{Lt}$ & Rt temporal (nonlesional) & 12 \\
\hline 12 & 48 & $\mathrm{~F}$ & Rt & $\mathrm{Lt}$ & Rt temporal (nonlesional) & 8 \\
\hline 13 & 27 & M & Rt & $\mathrm{Lt}$ & Rt occipital (lesional) & 8 \\
\hline
\end{tabular}

$\mathrm{fMRI}=$ functional MRI.

based (J.M.), frameless (D.N.), iSYS1 (S.W. and C.D.), and Neuromate (M.T.) robotic implantation methods.

\section{Methods \\ Patients}

We included 13 consecutive patients who underwent manually determined planning of electrode placement and surgical implantation between July 2015 and October 2016. Informed consent was obtained from all patients before their inclusion in the study. The National Research Ethics Service Committee London approved this study. Patient demographics are summarized in Table 1.

The case of each patient had been discussed by a multidisciplinary team (MDT) that consisted of epileptologists, neurosurgeons, neuropsychologists, neuropsychiatrists, and neuroradiologists. From the noninvasive presurgical evaluation, the team agreed on the hypothesized EZ and determined the requirement for invasive EEG recording. Patients who required subdural grid implantation were excluded from the study. Members of the MDT also agreed on regions for sampling for SEEG and generated a list of brain regions that required sampling. Before final approval by the MDT, manual plans were then created by a consulting neurosurgeon who had subspecialty expertise in epilepsy surgery.

\section{Multimodal Imaging}

MRI was performed on a GE 3-T MR750 scanner with a 32-channel head coil. A coronal 3D T1-weighted magnetization-prepared rapid-acquisition gradient echo scan was performed with a field of view (FOV) of $224 \times 256 \times 256$ $\mathrm{mm}$ (anterior to posterior, left to right, inferior to superior, respectively) and an acquisition matrix of $224 \times 256 \times$ 256 , for a voxel size of 1-mm isotropic resolution (TE/TR/ TI 3.1/7.4/400 msec; flip angle $11^{\circ}$; parallel imaging acceleration factor 2). 3D FLAIR scans were acquired with a 3D fast-spin echo sequence with variable flip-angle read- out (CUBE) with the same FOV and acquisition matrix, for a 1-mm isotropic resolution (TR/TI/TE 6200/1882/137 msec; echo train length 150; parallel imaging acceleration 2 [along both the in-plane and through-plane phase-encoding axes]). Vascular imaging comprised postgadolinium T1-weighted and phase-contrast MR angiography (MRA) and MR venography (MRV) scans. The axial postgadolinium T1-weighted scan was acquired with a fast spoiled gradient echo sequence with a FOV of $256 \times 256 \times 224$ $\mathrm{mm}$ and an acquisition and reconstruction matrix of $256 \times$ $256 \times 224\left(\mathrm{TE} / \mathrm{TR} 3.1 / 7.4 \mathrm{msec}\right.$; flip angle $\left.11^{\circ}\right)$. MRA and MRV were performed using a 3D phase-contrast sequence with a FOV of $220 \times 220 \times 148.8 \mathrm{~mm}$ and an acquisition matrix of $384 \times 256 \times 124$, for a reconstructed voxel size of $0.43 \times 0.43 \times 0.60 \mathrm{~mm}$ (flip angle $8^{\circ}$; parallel imaging acceleration factor 2). To highlight the arteries, MRA was performed with a velocity encoding of $80 \mathrm{~cm} / \mathrm{second}$ (TE/ TR 4.0/9.3 msec). For sensitivity to the venous circulation, the MRV was performed with a velocity encoding of 15 $\mathrm{cm} / \mathrm{second}$ (TE/TR 4.8/26.4 msec), fat suppression, and a saturation band inferior to the FOV.

\section{Manual Planning}

Manual plans were generated using volumetric T1weighted gadolinium-enhanced images as the reference image on which MRV images were coregistered, and vessels were extracted using a previously described tensor voting framework algorithm. ${ }^{30}$ Entry and target points were placed manually using axial, coronal, and sagittal reconstructions, and trajectories were checked using the "probe's-eye" function. A 3D model of the cortical surface was used to ensure that entry points were on the crown of gyri.

\section{EpiNav}

\section{Data Processing and Model Generation}

EpiNav is a software platform that allows multimodal image coregistration, vessel segmentation, 3D model gen- 
eration, and manual and automated electrode planning. T1-weighted magnetization-prepared rapid-acquisition gradient echo sequences were submitted for whole-brain parcellation (geodesic information flows) from which cortical, gray matter, and sulcal models were generated., ${ }^{6,20}$ Preoperative CT scans were used to generate skull models, which then were modified to prevent entry through the contralateral hemisphere, face, ear, posterior fossa, and skull base.

The technical aspects of the CAP algorithm used in this study were described previously. ${ }^{23}$ In brief, the user defines target points as ROIs for electrode sampling, which can be done by typing the name of a structure (e.g., right amygdala) or clicking on the ROI of the brain-parcellation image. The entry ROI can be specified if a superficial target is also required (e.g., entry through the motor cortex to target the supplementary motor area), but it is not obligatory. In this study, the same target points and, if specified, entry points were selected based on the requirements of the SEEG MDT planning meeting. The user defines a maximum electrode length $(90 \mathrm{~mm}$ was applied for all electrodes) and a maximum drilling angle $\left(25^{\circ}\right.$ orthogonal to the skull). The CAP algorithm then removes any potential electrode trajectories that do not adhere to length and angle constraints before ensuring that the trajectories pass through the skull model to the target ROI. If an entry ROI is defined, trajectories that do not pass through this ROI will be removed also. Then, the remaining trajectories are checked to ensure that they do not collide with a critical structure such as a blood vessel or sulcus. A minimum distance from vessels can be set as a safety margin by the user ( $3 \mathrm{~mm}$ was used for all electrodes in this study). The electrode trajectories that satisfy the requirements are then stratified based on risk, which is calculated as a function of the cumulative distance from vessels along the whole trajectory, optimized for gray matter contact and adjusted to avoid conflicts with other electrode trajectories. The electrode trajectories then are presented for review by the using the probe's-eye function linked to the orthogonal planes. Then, the resulting electrode trajectories are iterated by using either the "next entry" or "next target" button until a feasible electrode trajectory is chosen by the user (Fig. 1).

\section{Risk Metric Calculation}

EpiNav provides a graphic of the minimum distance from vasculature along the length of the electrode and a quantitative representation of the following safety metrics for both manually and CAP-determined electrode-placement plans, which were used for comparison: 1) electrode length, 2) drilling angle, 3) risk, 4) gray/white mattersampling ratio, and 5) the minimum distance from vessels.

\section{External Validation}

Five independent external raters who were neurosurgeons with expertise in performing electrode implantations for SEEG performed the external validation. The external raters had a range of experience with different implantation techniques, including frame-based (J.M.), frameless (D.N.), iSYS1 (S.W. and C.D.), and Neuromate
(M.T.) robotic implantation methods. A prospective power calculation based on a pilot study in which 14 electrodes from 2 patients were rated by a surgeon (M.T.) revealed that 24 electrodes were required to detect an absolute difference in risk of 0.2 assuming an SD of 0.3 and a power of 0.90 to achieve a 2-tailed significance level of $p=0.05$. To account for a potential clustering effect, a total of 13 patients were recruited. All raters appraised the same 2 pairs of plans ( $n=32$ electrodes) to assess interrater variability and another 3 or 4 sets of paired plans $(n=34-41$ electrodes) independently. All raters were blinded to the electrode-trajectory-generation method and were asked to provide ratings of the entry, trajectory, and target feasibility for paired manually and CAP-determined electrodes. Raters were asked to rate the feasibility of each trajectory based on their current implantation practice. Given that the sampling region suitability had been approved by the MDT based on the noninvasive presurgical evaluation, the raters were asked to comment only on the surgical feasibility of electrode implantation.

\section{Statistical Evaluation}

Risk metrics for manually and CAP-determined electrode placement were confirmed to have a normal distribution through the Shapiro-Wilks test $(\mathrm{p}>0.05)$. A paired Student t-test was used for manually and CAP-determined electrode-placement plan comparisons. Clustering of electrodes within patients was assessed by using a patientspecific random-effects model (model 1) and the possible difference between surgeons by using a fixed-effect model (model 2). A generalized likelihood ratio test was performed to compare models 1 and 2 , which resulted in a $\mathrm{p}$ value of 0.151 , indicating that insufficient evidence was found to suggest a significant difference between surgeons with regard to feasibility ratings. Feasibility ratings of electrode-placement plans generated from the manual and CAP methods were compared using the McNemar test, and odds ratios were calculated.

\section{Results}

Thirteen consecutive patients who underwent implantation of 116 electrodes for SEEG were included in the study. Manually determined plans were not provided for 12 electrodes out of concern for reaching specified targets safely; however, trajectories for these electrodes were generated with CAP. As such, paired results for the safety metric comparison were available for 104 electrodes (Fig. 2 and Table 2).

\section{Interrater Variability}

The surgeons rated each electrode for feasibility of the entry point, trajectory, and target point. If all 3 ratings were deemed feasible, the electrode was deemed feasible as a whole. All surgeons initially rated the same 2 pairs of plans (18 from CAP and 14 from the manual method) to assess interrater variability. A generalized likelihood ratio produced a test statistic of 6.72 . When compared with the quantiles of chi-square distribution with 4 degrees of freedom, a $\mathrm{p}$ value of 0.11 was obtained, which implies that insufficient evidence was found to suggest a difference in 

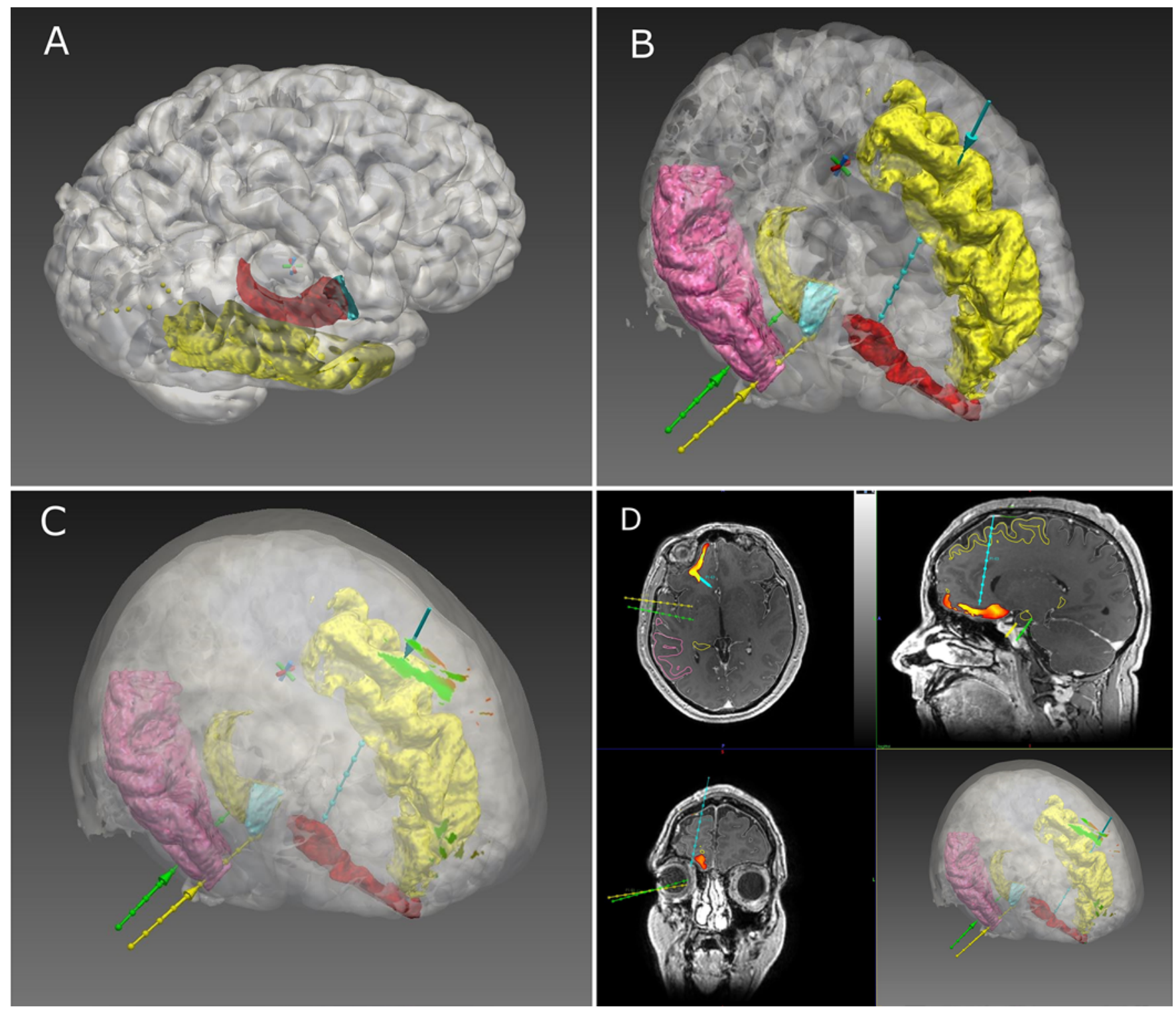

FIG. 1. Computer-assisted determination of electrode-placement workflow. A: Using the EpiNav strategy, module ROls are segmented automatically from the parcellation image. In this example, the cortex (white) is semitransparent to enable visualization of the underlying middle temporal gyrus (yellow), amygdala (blue), and hippocampus (red). B: Entry and target points for the electrodes within the strategy are generated automatically based on the safety metrics defined by the user. Electrodes are indicated in the right amygdala (yellow trajectory), right anterior hippocampus (green trajectory), and right posterior mesial orbitofrontal (blue trajectory). C: A surface risk heat map on the scalp was generated for the mesial orbitofrontal electrode as an example to show the safety of potential trajectory entry points. D: Orthogonal and 3D views showing the target risk heat map was generated for the mesial orbitofrontal electrode as an example to show safe trajectory target points in the orthogonal planes. Note that only 3 electrodes are shown for clarity. A probe's-eye view (not shown) can then be linked to the orthogonal planes for further assessment of the electrode trajectories. Figure is available in color online only.

surgeon ratings. The remaining 98 electrode-rating pairs were then pooled.

\section{Feasibility of Electrode Trajectories}

Based on external independent ratings, both the manually and CAP-determined electrode placements were rated as feasible in $42.8 \%$ of the cases. CAP provided feasible electrode placement in $19.4 \%$ of the cases, whereas manual planning generated feasible electrode placement in $26.5 \%$ of the cases in which the alternative placementgeneration method was not feasible. In $11.2 \%$ of the cases, the CAP- and manually determined electrode plans both were rated as not feasible (Table 3 ).

\section{Time to Generate Plans}

The manually and CAP-determined electrode-placement plans were generated using EpiNav, which requires multimodal images to be coregistered and segmentation 

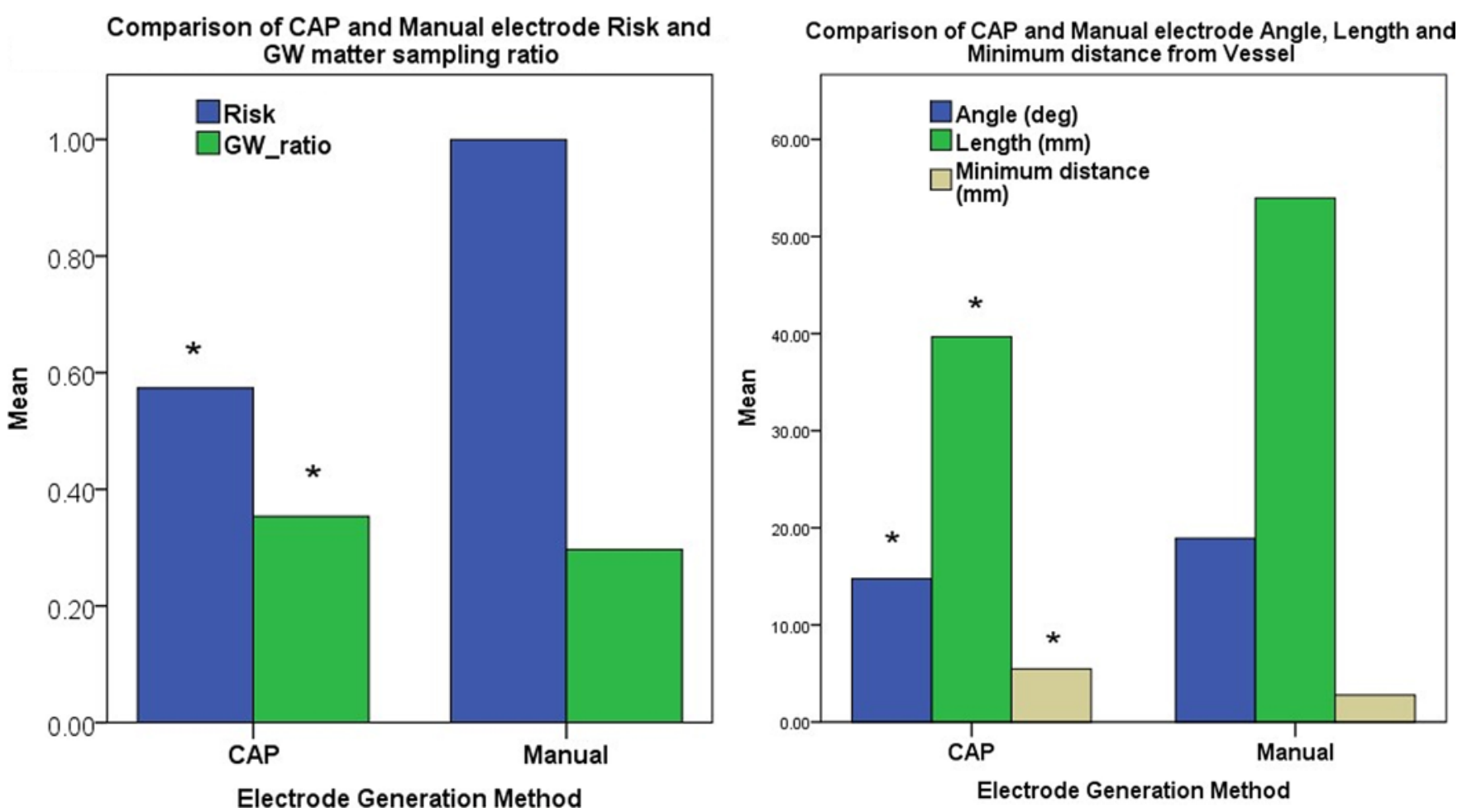

FIG. 2. Left: Comparison of risks and gray/white matter (GW)-sampling ratios between CAP and manual planning for electrode placement showing a statistically significant reduction in risk and improvement in GW sampling ratios. Right: Comparison of trajectory angles, lengths, and minimum distances from segmented vessels showing a statistically significant reduction in electrodetrajectory length and drilling angle and increase in the minimum distance from vasculature with the use of CAP compared with manual planning. ${ }^{*} p<0.01$. Figure is available in color online only.

of vascular, sulcal, and gray matter models before electrode planning. This time was common to both methods and, depending on the number of images, can take up to 60 minutes. Both CAP and manual planning require the generated electrode-placement plans to be checked using the probe's-eye and orthogonal views to ensure that they are suitable, which takes approximately 2 minutes per trajectory. Time taken to generate the plans using the manual method varied from 2 to 4 hours, and the computational time for CAP varied from 34 to 120 seconds.

\section{Discussion}

\section{Previous Studies of CAP}

CAP for surgical interventions provides the potential to automate time-consuming tasks and optimize clinically significant parameters to improve the safety and efficacy of surgical interventions. Unlike human users, CAP systems provide reliable and reproducible results regardless of the institution or team that provides the intervention. CAP algorithms, however, are only as good as the information provided to them. As a result, rigorous quality assurance is required for the imaging acquisition, postprocessing, and model segmentation used to generate CAP-determined electrode-placement plans. The success of epilepsy surgery depends on the detection and safe resection of the $\mathrm{EZ}$, which is the minimum region of brain that is required to be resected or ablated to result in sustained freedom from seizures. In cases in which the presumed seizureonset zone cannot be defined accurately because of a discrepancy or lack of concordance in the noninvasive presurgical evaluation (imaging studies, scalp EEG, and neuropsychological investigations), invasive EEG via subdural grid/strip implantation or SEEG is indicated. SEEG investigations involve the stereotactic placement of electrodes within predefined brain structures to enable recording of the spatial and temporal evolution of interictal and ictal activities. This recording is used subsequently to guide surgical resection margins and functional cortical mapping. Here, we describe a multimodal imaging platform for automated electrode planning that enables multiple electrode trajectories to be implanted into anatomically defined structures while avoiding conflicts with other electrodes, maintaining a user-defined safety margin from cerebral vasculature, increasing cumulative distance from vessels, preventing crossing of sulcal pial boundaries, and maximizing gray matter sampling while reducing intracerebral electrode length and drilling angles.

Initial studies of CAP in neurosurgery were described in the 1980s for stereotactic intracranial biopsies., ${ }^{72}$ The system described by Davis et al. ${ }^{7}$ enabled the coregistration of preoperative MRI scans with digital subtraction angiography (DSA) and a CT scan performed once patients were placed in a stereotactic frame. The target points for the biopsies were placed by the surgeon manually, and the computer system automatically calculated the stereo- 
TABLE 2. Risk metric comparison between CAP and manual planning results

\begin{tabular}{|c|c|c|c|}
\hline Metric & CAP* & $\begin{array}{c}\text { Manual } \\
\text { Planning* }\end{array}$ & $\begin{array}{c}p \\
\text { Value† }\end{array}$ \\
\hline Length (mm) & $39.8 \pm 14.9$ & $54.0 \pm 14.7$ & 0.001 \\
\hline Drilling angle $\left({ }^{\circ}\right)$ & $14.8 \pm 5.8$ & $18.9 \pm 9.0$ & 0.001 \\
\hline Gray matter-sampling ratio & $0.35 \pm 0.2$ & $0.30 \pm 0.16$ & 0.007 \\
\hline $\begin{array}{l}\text { Minimum distance from vessel } \\
(\mathrm{mm})\end{array}$ & $5.4 \pm 3.0$ & $2.8 \pm 1.9$ & $<0.001$ \\
\hline Risk & $0.57 \pm 0.39$ & $1.00 \pm 0.60$ & 0.001 \\
\hline
\end{tabular}

tactic coordinates. Potential trajectories were then simulated on anterior-posterior and lateral projections. Davis et al. provided results from 447 biopsies performed in 439 patients for both supratentorial and infratentorial targets over a 5-year period; a histological diagnosis was achieved for $99 \%$ of the patients, and a clinically significant hemorrhage occurred in $<1 \%$ ( 3 of 439) of them. The next significant advance in CAP was the introduction of 3D reconstruction of the cortex to enable surgeons to choose the most appropriate surgical trajectory for the resection of supratentorial mass lesions. ${ }^{11}$ Giorgi et al. ${ }^{12}$ used this process to plan a transfrontal approach as an alternative to the transcallosal approach for intraventricular lesions and thereby prevented the neuropsychological complications related to partial corpus callosotomy. Another iteration of this system was used to enable manual segmentation of lesions and improve the distinction between normal brain structures. Zamorano et al. ${ }^{29}$ described the Wayne State University hardware and software configuration, which in addition to preplanning surgical approaches could also be used intraoperatively with a neuronavigation system to track instruments in real time relative to the patient's head. The Neuroplanner software also integrated multiple brain atlases within a CAP system for functional neurosurgical procedures such as thalamotomy, pallidotomy, and deep brain stimulation (DBS). ${ }^{19}$ This system reduced operative time, improved targeting accuracy, reduced the number of surgical complications, and lowered the overall cost of the procedure. The use of clinical information to build on and guide further surgery was described by Guo et al., ${ }^{13}$ who developed probabilistic functional maps to guide targeting of the subthalamic nucleus for DBS. Here, the automated CAP-determined targets and trajectories in 10 patients were compared with those developed by an experienced stereotactic neurosurgeon. The average distance between the CAP- and manually determined target points was, on average, $<2 \mathrm{~mm}$. The incorporation of trajectory risk was used by Vaillant et al. ${ }^{27}$ based on whether a particular trajectory intersected a critical brain structure and the relative weighting given to the importance of that structure. Given that the major complications of stereotactic electrode placement include hemorrhage and inaccuracy of structure targeting, the inaccuracy of the implantation method also requires consideration. ${ }^{5,28}$ Therefore, just calculating whether an electrode conflicts with a critical
TABLE 3. External blinded ratings of electrode feasibility

\begin{tabular}{cccc}
\hline \multirow{2}{*}{ CAP } & \multicolumn{2}{c}{ Manual Planning Feasible } & \\
\cline { 2 - 3 } Feasible & Yes & No & Total \\
\hline Yes & $42.8(42)$ & $19.4(19)$ & $62.2(61)$ \\
\hline No & $26.5(26)$ & $11.2(11)$ & $37.8(37)$ \\
\hline Total & $69.4(68)$ & $30.6(30)$ & $100(98)$ \\
\hline
\end{tabular}

Values are presented as the percentage (number) based on 98 electroderating pairs.

structure (such as an intracerebral vessel) and how close the electrode passes to it along its trajectory are not sufficient. Cardinale et al. ${ }^{4}$ introduced the concept of a minimum safety margin when planning electrode placements for SEEG based on the accuracy of the implantation method being used, and they calculated it by using the following equation: planning safety margin $=$ electrode radius + mean implantation error +3 SDs.

Based on this equation, a minimum distance of $3 \mathrm{~mm}$ was recommended so that $99 \%$ of electrodes will fall within this safety margin. Once a minimum planning distance is set, risk for candidate trajectories can be calculated and represented as a heat map on the cortical surface. ${ }^{1,16,21}$ The calculation of risk, however, is based on the accuracy and completeness of the segmentation of critical structures. In the case of cerebral vasculature, a number of different vessel segmentation methods, including gadolinium-enhanced MRI, MRV, MRA, time-of-flight MRI, and DSA, have been used. ${ }^{5}$ The gold-standard method is DSA, but it entails an invasive procedure and radiation exposure. Noninvasive techniques visualize fewer segmented vessels, but unclear is whether this limitation is clinically significant and whether there is a minimum vessel size that must be avoided. A simple weighting based on vessel size might not be appropriate, because multiple factors, such as stylet design, vessel tethering, and the vessel wall (artery versus vein), also have an effect on the likelihood of hemorrhage. ${ }^{3}$ After reviewing complications associated with the placement of electrodes for DBS, Elias et al. ${ }^{9}$ described a hemorrhagic complication rate of $10 \%$ in patients when an electrode crossed a sulcus and an intraventricular hemorrhage rate of 5\% after ventricular penetration. Bériault et al..$^{1,2}$ presented a CAP algorithm that avoided segmented vasculature, critical neurological structures, ventricles, and sulci and did not allow crossing of the midline, which provided qualitative safety metrics for each trajectory. Trope et al. ${ }^{26}$ added tractography and fMRI data and found that the presentation of multimodal information to the surgeon resulted in a change in trajectory for intracranial biopsies in $85 \%$ of cases. Shenai et al. ${ }^{22}$ described the use of CAP for the stereotactic placement of depth electrodes within the amygdalohippocampal complex in patients with epilepsy. The system resulted in an additional electrode contact being inserted within the target structure. De Momi et al. ${ }^{8}$ described an automated system for the placement of multiple electrodes for SEEG in which the entry and target points were "roughly" selected and they considered the drilling angle to the skull and distance from other electrodes when they calculated the 
optimal trajectories. Clinical validation of 26 electrodes in 3 patients was assessed by 4 blinded neurosurgeons, and feasible electrode placements were planned in $86 \%$ of the cases; in $30 \%$ of the cases, these system-generated electrode placements were preferred to those planned manually. It should be noted that their CAP resulted in a distance from vessels along the first $25 \mathrm{~mm}$ of the trajectory that was significantly greater than the distance of those planned manually.

\section{Improvements From Previous Work}

We previously described use of the EpiNav software platform for automated placement of electrodes for SEEG based on a user-defined target and the aforementioned constraints. ${ }^{18}$ We subsequently improved on this work by allowing entire anatomical structures to be selected as the target point based on whole-brain parcellation. This process, therefore, enables the safest target within the anatomical structure of interest to be selected, because manually placed targets might not represent the safest option. Furthermore, to improve the feasibility of electrode-placement plans and to account for different surgical preferences, we allowed the user to iterate through risk-stratified CAP-generated electrodes. The development of a next-target or next-entry function enabled users to iterate through computed trajectories until they were satisfied with the trajectory. In line with our previous work, we have shown that targeting whole structures, instead of specific target points, results in safety metrics that are better than those found with manually generated plans.

\section{External Validation of CAP}

To provide external validation of the CAP-determined trajectories, 116 paired manually and CAP-determined electrode-placement plans for 13 patients were rated by neurosurgeons from external institutions who had expertise in SEEG. The manually determined electrodeplacement plans presented to the raters had already been implemented, and no hemorrhages (clinically or nonclinically significant) had occurred, so they were, by definition, feasible. Interesting to note is that $69.4 \%$ of manually determined implantation plans were rated as feasible by the external raters, which reflects the variation in individual surgeon practices and preferences depending on the implantation method used. Raters were asked to rate the feasibility of the trajectories based on their individual practices and whether they would be prepared to implant the electrodes using these trajectories themselves. It would be expected, therefore, for the raters to use different safety margins and heuristics, such as crossing of sulci, when assessing the trajectories. CAP-determined trajectories were deemed feasible in $62.2 \%$ of the cases, and it generated feasible electrodes in $19.2 \%$ of the cases in which manual plans were considered not feasible. When externally rated, CAP generates clinically feasible electrode-placement plans that are no less feasible than those produced manually. To our knowledge, this is the first study in which both manually and CAP-determined electrode-placement plans have been rated by blinded external raters to provide a more methodologically robust comparison between the 2 implantation methods.

\section{Limitations}

The main methodological limitation of this study is that it was retrospective in nature. Retrospective comparisons provide the potential for bias when the comparison data set is generated. Given that the CAP data were generated in an automated fashion, the effects of bias are likely to be minimal many months after the manual plans but cannot be excluded completely. A prospective validation study is currently underway.

MRV vessel segmentations were used to generate both the CAP- and manually determined electrode trajectories. The gadolinium-enhanced T1-weighted sequences were used as the reference image against which raters assessed trajectory feasibility. Gadolinium-enhanced T1-weighted sequences highlight a number of vessels that are not possible to segment with MRV. As such, one would expect manual planning to be favored over CAP. Significant heterogeneity exists between the vessel-segmentation methods used within European and North American epilepsy surgery centers. Although DSA is regarded by many as the gold standard, it is in itself an invasive investigation that carries risk and exposes the patient to radiation. Given that DSA was not the standard of care in our institution at the time of manual electrode-implantation planning, we were unable to assess the effect of DSA on CAP-determined trajectories. A potential future improvement of CAP would be to use DSA or multimodal MR vessel segmentations.

Sulcal models used for CAP-determined electrode trajectories are based on brain parcellation and the ability to segment CSF. The presence of CSF below the level of the gyrus is then considered to be within a sulcus, which is used as a region for exclusion during generation of the CAP-determined electrode trajectories. CSF-based sulcal models are not optimal for young patients, because the majority of their sulci do not have visible CSF within them, and their sulci are "potential" as opposed to actual spaces. Further improvement in sulcal model generation is likely to lead to improved safety in using CAP-determined electrode trajectories.

EpiNav has an integrated export function to enable planned trajectories to be exported seamlessly to the S7 StealthStation (Medtronic, Inc.). The software does not currently export to other neuronavigation systems seamlessly, which could reduce the number of potential users of the software, especially in the developing world. The software runs on most Windows PCs that contain a suitable graphics card. EpiNav was developed at University College London and is not commercial software. We are disseminating it free of charge for use at collaborating centers after appropriate local research ethics committee approval.

\section{Conclusions}

Here, we provide the results of a retrospective validation study of CAP for the placement of electrodes for SEEG in patients with drug-resistant focal epilepsy. CAP electrodeplacement plans overall resulted in an improved risk profile, increased minimum distance from vessels, shorter intracranial length, increased gray matter sampling, and lower drilling angles. CAP-determined electrode trajectories were assessed by blinded external raters as feasible in 
$62.2 \%$ of cases compared with $69.4 \%$ of manually generated trajectories and were also found to be feasible when manually planned electrodes were not deemed feasible in $19.4 \%$ of cases. CAP is a valuable tool that can be used as a first-line method of electrode-trajectory generation. The electrodes can then be reviewed by the surgeon, who can iterate through alternative CAP-generated trajectories or replan electrode placements manually when the CAP-determined trajectories are deemed to not be feasible. Given that electrode trajectories can be generated by CAP in a fraction of the time it takes to determine them manually, it is likely to reduce the planning burden while ensuring improved safety metrics.

\section{Acknowledgments}

This work was supported by the Wellcome Trust (innovation grant 106882) and the National Institute for Health Research University College London Hospitals Biomedical Research Centre. G.P.W. was supported by a Medical Research Council clinician scientist fellowship (MR/M00841X/1).

\section{References}

1. Bériault S, Al Subaie F, Collins DL, Sadikot AF, Pike GB: A multi-modal approach to computer-assisted deep brain stimulation trajectory planning. Int J Comput Assist Radiol Surg 7:687-704, 2012

2. Bériault S, Al Subaie F, Mok K, Sadikot AF, Pike GB: Automatic trajectory planning of DBS neurosurgery from multimodal MRI datasets. Med Image Comput Comput Assist Interv 14:259-266, 2011

3. Brunenberg EJL, Vilanova A, Visser-Vandewalle V, Temel Y, Ackermans L, Platel B, et al: Automatic trajectory planning for deep brain stimulation: a feasibility study. Med Image Comput Comput Assist Interv 10:584-592, 2007

4. Cardinale F, Cossu M, Castana L, Casaceli G, Schiariti MP, Miserocchi A, et al: Stereoelectroencephalography: surgical methodology, safety, and stereotactic application accuracy in 500 procedures. Neurosurgery 72:353-366, 2013

5. Cardinale F, Pero G, Quilici L, Piano M, Colombo P, Moscato A, et al: Cerebral angiography for multimodal surgical planning in epilepsy surgery: description of a new threedimensional technique and literature review. World Neurosurg 84:358-367, 2015

6. Cardoso MJ, Modat M, Wolz R, Melbourne A, Cash D, Rueckert D, et al: Geodesic information flows: spatially-variant graphs and their application to segmentation and fusion. IEEE Trans Med Imaging 34:1976-1988, 2015

7. Davis DH, Kelly PJ, Marsh WR, Kall BA, Goerss SJ: Computer-assisted stereotactic biopsy of intracranial lesions in pediatric patients. Pediatr Neurosci 14:31-36, 1988

8. De Momi E, Caborni C, Cardinale F, Casaceli G, Castana L, Cossu M, et al: Multi-trajectories automatic planner for StereoElectroEncephaloGraphy (SEEG). Int J Comput Assist Radiol Surg 9:1087-1097, 2014

9. Elias WJ, Sansur CA, Frysinger RC: Sulcal and ventricular trajectories in stereotactic surgery. J Neurosurg 110:201207, 2009

10. Fisher RS, Acevedo C, Arzimanoglou A, Bogacz A, Cross JH, Elger CE, et al: ILAE official report: a practical clinical definition of epilepsy. Epilepsia 55:475-482, 2014

11. Giorgi C, Broggi G, Casolino D, Franzini A, Pluchino F: Computer assisted analysis of neuroradiological data in planning neurosurgical procedures. J Neurosurg Sci 33:19-22, 1989

12. Giorgi C, Casolino SD, Franzini A, Servello D, Passerini A, Broggi G, et al: Computer-assisted planning of stereotactic neurosurgical procedures. Childs Nerv Syst 5:299-302, 1989

13. Guo T, Parrent AG, Peters TM: Automatic target and trajectory identification for deep brain stimulation (DBS) procedures. Med Image Comput Comput Assist Interv 10:483490, 2007

14. Keezer MR, Sisodiya SM, Sander JW: Comorbidities of epilepsy: current concepts and future perspectives. Lancet Neurol 15:106-115, 2016

15. Mullin JP, Shriver M, Alomar S, Najm I, Bulacio J, Chauvel $P$, et al: Is SEEG safe? A systematic review and meta-analysis of stereo-electroencephalography-related complications. Epilepsia 57:386-401, 2016

16. Nowell M, Rodionov R, Zombori G, Sparks R, Rizzi M, Ourselin S, et al: A pipeline for 3D multimodality image integration and computer-assisted planning in epilepsy surgery. J Vis $\operatorname{Exp}(\mathbf{1 1 1}): 53450,2016$

17. Nowell M, Rodionov R, Zombori G, Sparks R, Winston G, Kinghorn J, et al: Utility of 3D multimodality imaging in the implantation of intracranial electrodes in epilepsy. Epilepsia 56:403-413, 2015

18. Nowell M, Sparks R, Zombori G, Miserocchi A, Rodionov R, Diehl B, et al: Comparison of computer-assisted planning and manual planning for depth electrode implantations in epilepsy. J Neurosurg 124:1820-1828, 2016

19. Nowinski WL, Yang GL, Yeo TT: Computer-aided stereotactic functional neurosurgery enhanced by the use of the multiple brain atlas database. IEEE Trans Med Imaging 19:62-69, 2000

20. Prados F, Cardoso MJ, Burgos N, Gandini Wheeler-Kingshott CAM, Ourselin S: NiftyWeb: web based platform for image processing on the cloud, presented at the ISMRM 24th Annual Meeting \& Exhibition, 2016. (http://discovery. ucl.ac.uk/1476510/1/Submit-niftyweb.pdf) [Accessed November 29, 2017] (Abstract)

21. Shamir RR, Tamir I, Dabool E, Joskowicz L, Shoshan Y: A method for planning safe trajectories in image-guided keyhole neurosurgery. Med Image Comput Comput Assist Interv 13:457-464, 2010

22. Shenai MB, Ross DA, Sagher O: The use of multiplanar trajectory planning in the stereotactic placement of depth electrodes. Neurosurgery 60 (4 Suppl 2):272-276, 2007

23. Sparks R, Vakharia V, Rodionov R, Vos SB, Diehl B, Wehner $\mathrm{T}$, et al: Anatomy-driven multiple trajectory planning (ADMTP) of intracranial electrodes for epilepsy surgery. Int J Comput Assist Radiol Surg 12:1245-1255, 2017

24. Sparks R, Zombori G, Rodionov R, Nowell M, Vos SB, Zuluaga MA, et al: Automated multiple trajectory planning algorithm for the placement of stereo-electroencephalography (SEEG) electrodes in epilepsy treatment. Int J Comput Assist Radiol Surg 12:123-136, 2017

25. Téllez-Zenteno JF, Hernández-Ronquillo L, Buckley S, Zahagun R, Rizvi S: A validation of the new definition of drug-resistant epilepsy by the International League Against Epilepsy. Epilepsia 55:829-834, 2014

26. Trope M, Shamir RR, Joskowicz L, Medress Z, Rosenthal G, Mayer A, et al: The role of automatic computer-aided surgical trajectory planning in improving the expected safety of stereotactic neurosurgery. Int J Comput Assist Radiol Surg 10:1127-1140, 2015

27. Vaillant M, Davatzikos C, Taylor RH, Bryan RN: A pathplanning algorithm for image-guided neurosurgery, in Troccaz J, Grimson E, Mösges R (eds): CVRMed-MRCAS'97. Berlin: Springer, 1997, pp 467-476

28. Vakharia VN, Sparks R, O'Keeffe AG, Rodionov R, Miserocchi A, McEvoy A, et al: Accuracy of intracranial electrode placement for stereoencephalography: a systematic review and meta-analysis. Epilepsia 58:921-932, 2017

29. Zamorano L, Jiang Z, Kadi AM: Computer-assisted neu- 
rosurgery system: Wayne State University hardware and software configuration. Comput Med Imaging Graph 18:257-271, 1994

30. Zuluaga MA, Rodionov R, Nowell M, Achhala S, Zombori G, Mendelson AF, et al: Stability, structure and scale: improvements in multi-modal vessel extraction for SEEG trajectory planning. Int J Comput Assist Radiol Surg 10:1227-1237, 2015

\section{Disclosures}

The authors report no conflict of interest concerning the materials or methods used in this study or the findings specified in this paper.

\section{Author Contributions}

Conception and design: Vakharia, Sparks, Rodionov, McEvoy,
Miserocchi, Ourselin, Duncan. Acquisition of data: Vakharia, Sparks, Vos, Dorfer, Miller, Nilsson, Tisdall, Wolfsberger, McEvoy, Miserocchi. Analysis and interpretation of data: Vakharia. Drafting the article: Vakharia, Duncan. Critically revising the article: Vakharia, Sparks, Rodionov, Vos, Dorfer, Miller, Nilsson, Tisdall, Wolfsberger, McEvoy, Miserocchi, Winston, O'Keeffe, Duncan. Reviewed submitted version of manuscript: Vakharia, Sparks, Rodionov, Vos, Dorfer, Miller, Nilsson, Tisdall, Wolfsberger, McEvoy, Miserocchi, Winston, O'Keeffe, Duncan. Approved the final version of the manuscript on behalf of all authors: Vakharia. Statistical analysis: Vakharia, O'Keeffe. Administrative/technical/material support: Sparks. Study supervision: Ourselin, Duncan.

\section{Correspondence}

Vejay N. Vakharia: University College London, Institute of Neurology, London, United Kingdom.v.vakharia@ucl.ac.uk. 